\title{
Effect of the PA-MSHA vaccine on septic serum-induced inflammatory response
}

\author{
XIANG-FENG LIU ${ }^{1}$, LI WANG $^{2}$, YI QU ${ }^{3}$, DE-WU ZHONG $^{1}$, XIONG-YING MIAO ${ }^{1}$ and HONG-LIANG YAO ${ }^{1}$ \\ Departments of ${ }^{1}$ General Surgery and ${ }^{2}$ Cardiothoracic Surgery, Second Xiangya Hospital, \\ Central South University; ${ }^{3}$ Hunan Provincial People's Hospital, \\ Changsha, Hunan 410011, P.R. China
}

Received November 1, 2012; Accepted February 14, 2013

DOI: $10.3892 / \mathrm{mmr} .2013 .1337$

\begin{abstract}
Sepsis is defined as a complex clinical syndrome caused by a serious infection followed by an amplified and deregulated inflammatory response. The complex syndrome is associated with a high rate of morbidity and mortality, despite substantial clinical advances. A vaccine derived from the outer membrane proteins of the Gram-negative bacteria Pseudomonas aeruginosa (PA-MSHA) has been demonstrated to exhibit immune modulatory properties. In the present study, the effect of the PA-MSHA vaccine on the inflammatory response induced by serum from septic patients in peripheral blood mononuclear cells was determined. It was observed that PA-MSHA pretreatment inhibits the production of septic serum-induced tumor necrosis factor- $\alpha$. In addition, PA-MSHA treatment increases interleukin-10 levels and promotes the generation of $\mathrm{CD}^{+}{ }^{+} \mathrm{CD} 25^{+} \mathrm{Foxp}^{+} \mathrm{T}$ cells. Thus, the results of the current study provide mechanistic insight relevant to the potential application of PA-MSHA in the treatment of sepsis.
\end{abstract}

\section{Introduction}

Sepsis is an complex clinical condition characterized by a dysregulated inflammatory response to infection resulting in multiorgan failure with a fatal outcome (1-4). Currently, sepsis accounts for $12 \%$ patients admitted to the ICU despite the use of antibiotics (5). Gram-negative sepsis is also one of the most common and serious post-operative complications in abdominal surgery $(6,7)$. Despite major improvements in antimicrobial therapies, up to now, even highly effective treatments

Correspondence to: Professor Hong-Liang Yao, Department of General Surgery, Second Xiangya Hospital, Central South University, 138 Renmin Road, Changsha, Hunan 410011, P.R. China E-mail: yhlscience2012@hotmail.com

Dr Li Wang, Department of Cardiothoracic Surgery, Second Xiangya Hospital, Central South University, 138 Renmin Road, Changsha, Hunan 410011, P.R. China

E-mail: wangliscience@gmail.com

Key words: Pseudomonas aeruginosa, septic serum, tumor necrosis factor- $\alpha$, interleukin-10, regulatory T cells for sepsis syndrome have not been able to eliminate mortality. In addition, the excessive utilization of antibiotics increases the risk of development of sepsis caused by drug-resistant bacteria, particularly Gram-negative bacteria (8). Therefore, novel approaches for the prevention and early treatment of sepsis are likely to reduce the incidence and mortality of the syndrome. To date, the pathogenesis of sepsis remains unknown. The current understanding of Gram-negative sepsis is that the syndrome is evoked by the endotoxin produced by microbes and an overwhelming innate inflammatory response to a microbial infection, which correlates with the excessive release of immune factors, including interleukin (IL)-1, IL-6 and tumor necrosis factor (TNF)- $\alpha(4,9)$. Thus, an effective therapy may be directed at inhibiting the release of immune factors and interrupting the cytokine cascade.

As potentinducers of immune tolerance, $\mathrm{CD} 4^{+} \mathrm{CD} 25^{+} \mathrm{Foxp} 3^{+}$ regulatory $\mathrm{T}$ cells (Tregs) are important for the control of the immune response and prevention of the development of excessive immune-induced tissue damage (10-12). Tregs exert a pronounced anti-inflammatory effect largely by the contact-mediated direct inhibition of effector $\mathrm{T}$ cells and secretion of immunosuppressive cytokines, including IL-10 and transforming growth factor (TGF)- $\beta$, subsequently suppressing inflammatory response-induced damage $(10,11)$. Various studies have documented that the proportions of certain Tregs are increased and the suppressive functions of Tregs are amplified following sepsis or acute insult (13-15), but the role of these changes remains unclear as the depletion of Tregs in a septic mouse model was found to produce variable conclusions between models, improving, enhancing or bearing no effect on mortality (16-18). However, the vital role of Tregs in regulating proinflammatory cytokine formation secondary to severe insults is well established.

Pseudomonas aeruginosa is an extracellular, Gram-negative bacteria that increases the proliferative response of normal adult peripheral blood lymphocytes (19). Immunization with a vaccine derived from the outer membrane proteins of $P$. aeruginosa has been demonstrated to induce high titers of serum IgG antibody in rabbits and humans (20). A P. aeruginosa vaccine has been found to have anti-infective and anti-inflammatory functions as an immune modulator. $P$. aeruginosa mannose-sensitive hemagglutinin (PA-MSHA) is a form of peritrichous MSHA fimbria P. aeru- 
ginosa, which was established by $\mathrm{Mu}$ (21). Heat-inactivated PA-MSHA is currently available as a vaccine and functions as an effective immune modulator via activation of the proliferation and differentiation of dendritic cells to increase antigen presentation (22). However, its anti-inflammatory effect is not well understood and other mechanisms may be involved in this role.

In the present study, PA-MSHA was identified to induce an increased proportion of $\mathrm{CD} 4{ }^{+} \mathrm{CD} 25^{+} \mathrm{Foxp} 3^{+} \mathrm{T}$ cells in peripheral blood mononuclear cells (PBMCs). In addition, the stimulation of healthy PBMCs with serum isolated from Gram-negative sepsis patients was found to initiate the release of inflammatory mediators, whereas the exposure of PBMCs to PA-MSHA promoted the release of the immunosuppressive factor, IL-10 and reduced the production of the pro-inflammatory cytokine, TNF- $\alpha$. The potential mechanisms of PA-MSHA in inhibition of the inflammatory response are also discussed.

\section{Materials and methods}

Subjects. The samples analyzed in this study were derived from healthy donors and sepsis patients, who were enrolled in an open-label randomized trial. Patients with Gram-negative sepsis $(n=6)$ were consecutively hospitalized at the ICU of the Second Xiangya Hospital of Central South University (CSU) within 1 week, and met the severe sepsis criteria following abdominal surgery. The study was approved by the human ethics committee of the Institute of CSU. Informed consent was obtained from all participants in the study.

Serum. To prevent platelet activation and phospholipase activity, blood samples were collected in EDTA-containing tubes. Serum was obtained from peripheral blood samples by centrifuging and was kept at $-80^{\circ} \mathrm{C}$ until experimental assays were performed.

Cell preparation and cell treatment. PBMCs were derived from fresh heparinized blood samples from healthy adult donors. PBMCs were isolated by density gradient centrifugation and cultured in RPMI-1640 medium supplemented with $10 \%$ heat-inactivated fetal bovine serum. PA-MSHA (donated by Professor XY Mu in $1 \mathrm{ml}$ aliquots, containing inactivated PA-MSHA strain, $1.8 \times 10^{9}$ ) used in this study was scale-cultured at $37^{\circ} \mathrm{C}$ for $24 \mathrm{~h}$, inactivated using a chemical method and purified by centrifuging. The septic serum was enriched by ultracentrifuging. The healthy PBMCs were treated with PA-MSHA $\left(5 \times 10^{8} / \mathrm{ml}\right)$ and then stimulated with septic serum ( $1 \mathrm{ml}$ ) for various times, as indicated. Total RNA and cell culture supernatant were collected for further analysis.

RNA isolation and real-time quantitative RT-PCR. PBMCs were stimulated with septic or normal serum, following exposure to PA-MSHA. Total RNA was isolated from the cells with TRIzol (Invitrogen Life Technologies, Carlsbad, CA, USA). Real-time quantitative RT-PCR was performed using iQ5 (Bio-Rad, Hercules, CA, USA). Analyses were performed with $20 \mu 1$ reaction volumes containing $10 \mu 12$ X SYBR ${ }^{\circledR}$ Premix Ex Taq (Takara Bio, Inc., Shiga, Japan), $0.4 \mu \mathrm{M}$ each primer, $1 \mu \mathrm{l}$ cDNA template and 8.2 $\mu \mathrm{l}$ deionized water. PCR amplifica- tions were performed using the following parameters: $95^{\circ} \mathrm{C}$ for $10 \mathrm{sec}$ and 40 cycles of $95^{\circ} \mathrm{C}$ for $5 \mathrm{sec}$ and $60^{\circ} \mathrm{C}$ for $30 \mathrm{sec}$. Melting curve analysis was also performed to exclude non-specific PCR products. All PCR products were confirmed by melting curve analysis to exclude the possibility of multiple products or incorrect product size. PCR analyses were conducted in triplicate for each sample. Primers used were as follows: GAPDH, forward AGAAGGCTGGGGCTCATTTG and reverse AGGGGCCATCCACAGTCTTC; TNF- $\alpha$, forward CCTGTGAGGAGGACGAAC and reverse CCTGTGAGGAGGACGAAC; IL-10, forward TGA GAACAGCTGCACCCACTT and reverse TCGGAGAT CTCGAAGCATGTTA.

Flow cytometry.PBMCswere treated with septicorhealthy serum, following treatment with PA-MSHA. Following this, the treated cells were stained with antibodies against FITC-conjugated CD4, APC-CD25 and PE-Foxp3 (BD Biosciences, San Jose, $\mathrm{CA}$, USA). The percentage of $\mathrm{CD} 4^{+} \mathrm{CD} 25^{+} \mathrm{Foxp} 3^{+}$cells was measured by flow cytometry (FACSCalibur; BD Biosciences) and blank and isotype controls were used to eliminate autofluorescence and non-specific fluorescence.

ELISA measurement of cytokines. Secretion of TNF- $\alpha$ and IL-10 was determined by ELISA. PBMCs were plated in 24-well plates and treated with PA-MSHA prior to stimulation with septic serum. Next, media were harvested for measurement of cytokines. ELISA was performed according to the manufacturer's instructions (R\&D Systems, Minneapolis, MN, USA).

Statistical analysis. Analysis of data was performed using the unpaired t-test. Data are presented as the mean \pm SD. Analysis was performed using SPSS 16.0 and $\mathrm{P}<0.05$ was considered to indicate a statistically significant difference.

\section{Results}

PA-MSHA vaccine reduces TNF- $\alpha$ levels. The $P$. aeruginosa vaccine is known to exhibit anti-inflammatory effects. In the present study, the effect of the PA-MSHA vaccine on septic serum-induced TNF- $\alpha$ expression was examined in PBMCs. Healthy PBMCs were exposed to PA-MSHA, followed by stimulation using septic serum. Following this, total RNA and cell culture supernatant were collected at various times and TNF- $\alpha$ expression levels were determined by real-time RT-PCR and ELISA. As demonstrated in Fig. 1A, septic serum stimulation significantly increased the production of TNF- $\alpha$ during all the incubation periods tested compared with the untreated cells. The TNF- $\alpha$ levels increased and reached peak levels after $12 \mathrm{~h}$ of septic serum stimulation and decreased subsequently. However, the PA-MSHA-pretreated cells were found to release significantly lower levels of TNF- $\alpha$ compared with the cells stimulated with septic serum only at each time point. Consistent with ELISA results, the real-time RT-PCR results in Fig. 1B also revealed that stimulation of PBMCs with septic serum led to marked increases in TNF- $\alpha$ mRNA levels compared with those in the untreated cells, while PA-MSHA treatment markedly attenuated the TNF- $\alpha$ expression (Fig. 1B). These results indicate that PA-MSHA suppresses the production of septic serum-induced TNF- $\alpha$. 
A

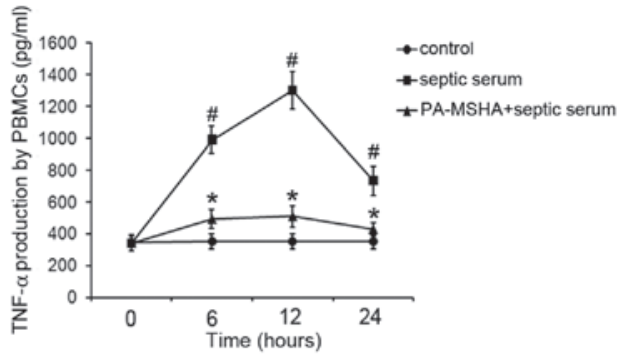

B

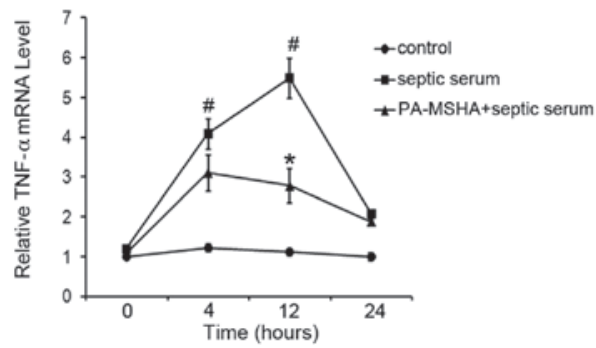

Figure 1. PA-MSHA reduces TNF- $\alpha$ expression. PBMCs were pretreated with PA-MSHA and then stimulated with septic serum for various times as indicated. Total RNA and cell culture supernatant were collected. (A) ELISA analyses revealed a significant increase in TNF- $\alpha$ production by PA-MSHA treated and non-treated cells following septic serum stimulation and the TNF- $\alpha$ levels in the PA-MSHA treated cells were significantly lower than those in the non-treated cells. (B) TNF- $\alpha$ mRNA levels were analyzed by real-time PCR and normalized against GAPDH mRNA. PCR results revealed that septic serum-stimulation markedly increased TNF- $\alpha$ mRNA levels and pretreatment attenuated the increase. ${ }^{~} \mathrm{P}<0.05$, vs. control; " $\mathrm{P}<0.05$, vs. septic serum-treatment. PA-MSHA, Pseudomonas aeruginosa mannose-sensitive hemagglutinin; PBMCs, peripheral blood mononuclear cells; TNF, tumor necrosis factor.

PA-MSHA vaccine increases IL-10 levels. Since PA-MSHA represses the induction of TNF- $\alpha$ by septic serum stimulation, the effect of the PA-MSHA vaccine on the levels of the anti-inflammatory factor, IL-10, was investigated. Using the septic serum-stimulated culture system, pretreatment of healthy PBMCs with PA-MSHA was observed to increase the induction of IL-10 levels by septic serum stimulation. As revealed in Fig. 2A, IL-10 levels in the supernatants of cells cultured in septic serum increased 2- or 3-fold compared with those in untreated cells, while PA-MSHA pretreatment was identified to result in a significant enhancement of the induction of IL-10 levels. IL-10 mRNA expression, as determined by RT-PCR, was consistent with the effects on protein concentration (Fig. 2B).

Effect of the PA-MSHA vaccine on the generation of $\mathrm{CD} 25^{+} \mathrm{Foxp} 3^{+}$Tregs. IL-10 is produced by cells of the immune lineage, mainly by macrophages and Tregs. Considering the significance of Tregs in sepsis, the effect of PA-MSHA on the generation of Tregs was determined. PBMCs were treated with PA-MSHA and then stimulated with septic serum. Post-stimulation (4 h), cells were collected and stained with antibodies against FITC-conjugated CD4, APC-CD25 and PE-Foxp3. Firstly, $\mathrm{CD} 4^{+} \mathrm{T}$ cells were sorted and then the proportion of $\mathrm{CD} 25^{+} \mathrm{Foxp}^{+} \mathrm{T}$ cells in the $\mathrm{CD} 4^{+} \mathrm{T}$ cells was determined. A significant increase in the percentage of Tregs was identified in the cells stimulated by septic serum [17.74\% (16.04-18.89\%)] compared with the control [9.04\% $(7.75-10.23 \%)]$. When the cells were pretreated with PA-MSHA, a more marked increase in the percentage of $\mathrm{CD} 25^{+} \mathrm{Foxp}^{+}$
A

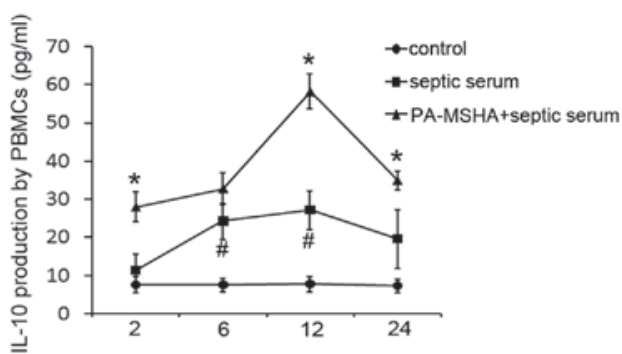

B

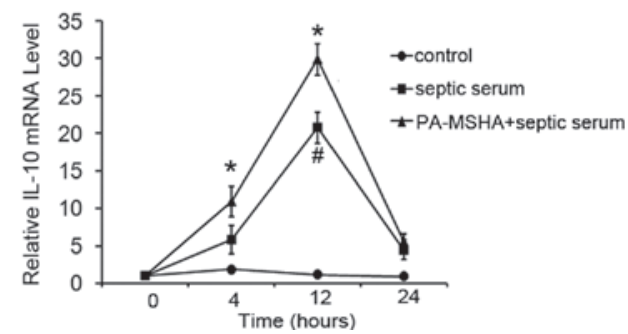

Figure 2. PA-MSHA vaccine increases IL-10 expression. PBMCs were pretreated with PA-MSHA vaccine and then stimulated with septic serum. Total RNA and cell culture supernatant were collected at indicated intervals following septic serum stimulation. (A) ELISA analyses revealed that PA-MSHA pretreatment markedly promoted septic serum-induced IL-10 release. (B) IL-10 mRNA was analyzed by real-time PCR and normalized against GAPDH mRNA. PCR results revealed that septic serum-stimulation increased IL-10 mRNA levels and the cells pretreated with PA-MSHA expressed higher levels of IL-10 compared with the cells treated with septic serum stimulation alone. ${ }^{\#} \mathrm{P}<0.05$, vs. control; ${ }^{*} \mathrm{P}<0.05$, vs. septic serum-treatment. PA-MSHA, Pseudomonas aeruginosa mannose-sensitive hemagglutinin; PBMCs, peripheral blood mononuclear cells; IL, interleukin.

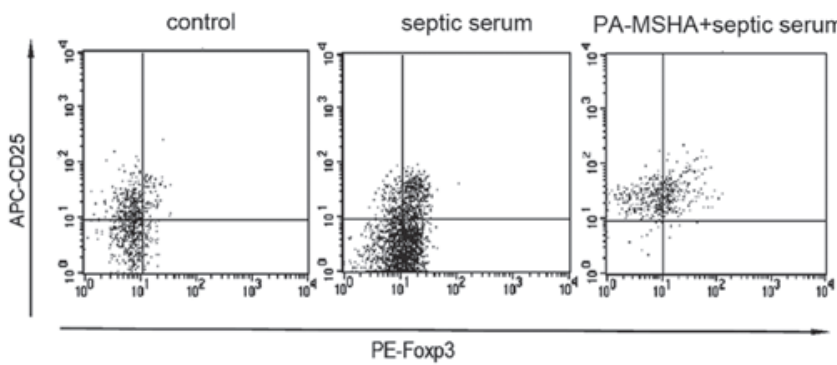

Figure 3. Percentages of $\mathrm{CD} 25^{+} \mathrm{Foxp} 3^{+} \mathrm{T}$ cells in $\mathrm{CD} 4^{+} \mathrm{T}$ cells by flow cytometry. PBMCs were pretreated with PA-MSHA vaccine and stimulated with septic serum. Post-stimulation $(4 \mathrm{~h})$, cells were collected and stained with FITC-conjugated CD4, APC-CD25 and PE-Foxp3 antibodies. CD4 ${ }^{+} \mathrm{T}$ cells were sorted and the proportion of $\mathrm{CD} 25^{+} \mathrm{Foxp}^{+}$cells in $\mathrm{CD}^{+}$cells was determined. Results reveal that septic serum stimulation increased the numbers of $\mathrm{CD} 25^{+} \mathrm{Foxp}^{+}$cells and PA-MSHA pretreatment significantly enhanced the induction. PA-MSHA, Pseudomonas aeruginosa mannose-sensitive hemagglutinin; PBMCs, peripheral blood mononuclear cells.

T cells [34.12\% (29.01-43.80\%)] was observed (Fig. 3). These results indicate that the PA-MSHA vaccine promotes the septic serum-stimulated induction of Tregs.

\section{Discussion}

The $P$. aeruginosa vaccine is currently widely used for anti-infective and -inflammatory purposes as an immune modulator. In a clinical study, we observed that the exposure of surgical wounds to a $P$. aeruginosa vaccine increased the 
rate of healing (unpublished data). To explore the potential mechanisms by which the $P$. aeruginosa vaccine represses the inflammatory response, changes in the cell immune and inflammatory factors produced by healthy PBMCs were analyzed following exposure to a $P$. aeruginosa vaccine, PA-MSHA, and stimulation by septic serum. The results indicated that exposure to PA-MSHA induced a more marked suppressive immune response, thereby inhibiting the release of pro-inflammatory cytokines.

Previous studies have demonstrated that PA-MSHA increases the antigen-presenting function by activating the proliferation and differentiation of dendritic cells, thus breaking down the complete and incomplete immunological tolerance, completely activating polyclonal $\mathrm{T}$ and $\mathrm{B}$ cells, increasing the number and proportion of $\mathrm{T}$ cells and $\mathrm{T}$ cell subgroups and stimulating the differentiation of intrinsic immunological active factors (22). The anti-inflammatory action of PA-MSHA indicates that the vaccine may also trigger a suppressive immune response to balance excessive inflammation. As a subset of T cells, Tregs may inhibit the activation of effector T-cell subsets in a contact-dependent manner or by producing inhibitory cytokines, including IL-10 and TGF- $\beta(10,11)$. As a transcription factor of Tregs, FoxP3 is a relatively specific marker of Tregs and also considered to be the key regulator (23). In the present study, stimulation of PBMCs with septic serum was demonstrated to induce an increased proportion of $\mathrm{CD} 4^{+} \mathrm{CD} 25^{+} \mathrm{Foxp} 3^{+}$ cells, while pretreatment of the cells with PA-MSHA elicited a more marked increase, indicating that Tregs may be involved in the anti-inflammatory role of PA-MSHA.

In vitro and in vivo studies in animals have indicated a protective role of IL-10 in atherosclerotic lesion formation and stability $(24,25)$. IL-10 is an important anti-inflammatory cytokine, which downregulates innate and adaptive immune responses and suppresses tissue inflammation and damage (26). IL-10 potently represses the inflammatory response largely through blocking the maturation of antigen-presenting cells, inhibiting the production of pro-inflammatory cytokines and directly suppressing the differentiation of $\mathrm{T}$ cells into effector subsets (27). IL-10 is known to be produced by various inflammatory cells, among them macrophages and Tregs. In the present study, the exposure of PBMCs to PA-MSHA was revealed to induce the suppressive inflammatory factor, IL-10, which may reduce the magnitude of the immune response against septic serum, thus minimizing consequent damage due to sepsis. In addition, the observation of enhanced IL-10 release by PBMCs pretreated with PA-MSHA is supported by the increased Treg numbers and may be explained by this increase. Thus, these observations indicate that PA-MSHA may induce the differentiation and development of Tregs and promote the increased production of IL-10, resulting in an alleviated inflammatory response.

Previous studies have revealed that in sepsis, the serum concentration of IL-10 is also increased. This suppressive factor functions to inhibit the release of TNF- $\alpha$, IL-1 $\beta$ and IL-6, and reduces the circulating concentrations of these cytokines. A number of the classical features of inflammation in sepsis are attributed to the actions of TNF- $\alpha$ (9). Serum concentrations of TNF- $\alpha$ correlate with mortality in specific types of human sepsis $(28,29)$. In the current study, PBMCs exposed to PA-MSHA were found to produce lower levels of TNF- $\alpha$ than cells which had not been pretreated, following stimulation with septic serum. This result indicates that PA-MSHA may reduce the release of the key cytokine TNF- $\alpha$ in sepsis. Considering the role of IL-10 in regulating the release of TNF- $\alpha$ in sepsis, we hypothesize that PA-MSHA may exert its anti-inflammatory role through promoting the development of Tregs, consequently suppressing the release of TNF- $\alpha$.

In summary, results of the present study indicate that PA-MSHA suppresses the inflammatory response during sepsis. PA-MSHA represses the key inflammatory cytokine, TNF- $\alpha$. This process may be mediated by the induction of Treg development and promotion of IL-10 production. Since the vaccine reveals inhibitory potential against the inflammatory response, PA-MSHA may be suitable for the prevention of early sepsis.

\section{Acknowledgements}

This study was supported by a grant from the Nature Scientific Foundation of Hunan Province (no. 2009FJ3186 and 2012Rs4021).

\section{References}

1. Bone RC, Balk RA, Cerra FB, et al: Definitions for sepsis and organ failure and guidelines for the use of innovative therapies in sepsis. The ACCP/SCCM Consensus Conference Committee. American College of Chest Physicians/Society of Critical Care Medicine. Chest 101: 1644-1655, 1992.

2. Warren HS: Strategies for the treatment of sepsis. N Engl J Med 336: 952-953, 1997.

3. Stone R: Search for sepsis drugs goes on despite past failures. Science 264: 365-367, 1994.

4. Hotchkiss RS and Karl IE: The pathophysiology and treatment of sepsis. N Engl J Med 348: 138-150, 2003.

5. Curns AT, Steiner CA, Sejvar JJ and Schonberger LB: Hospital charges attributable to a primary diagnosis of infectious diseases in older adults in the United States, 1998 to 2004. J Am Geriatr Soc 56: 969-975, 2008.

6. Fukazawa A, Yokoi Y, Kurachi K, et al: Implication of B lymphocytes in endotoxin-induced hepatic injury after partial hepatectomy in rats. J Surg Res 137: 21-29, 2007.

7. Garcea $\mathrm{G}$ and Maddern GJ: Liver failure after major hepatic resection. J Hepatobiliary Pancreat Surg 16: 145-155, 2009.

8. Wright AJ, Unger S, Coleman BL, Lam PP and McGeer AJ: Maternal antibiotic exposure and risk of antibiotic resistance in neonatal early-onset sepsis: a case-cohort study. Pediatr Infect Dis J 31: 1206-1208, 2012.

9. Lewis DH, Chan DL, Pinheiro D, Armitage-Chan E and Garden OA: The immunopathology of sepsis: pathogen recognition, systemic inflammation, the compensatory anti-inflammatory response and regulatory $\mathrm{T}$ cells. J Vet Intern Med 26: 457-482, 2012.

10. Sakaguchi S, Wing K, Onishi Y, Prieto-Martin P and Yamaguchi T: Regulatory T cells: how do they suppress immune responses? Int Immunol 21: 1105-1111, 2009.

11. Workman CJ, Szymczak-Workman AL, Collison LW, Pillai MR and Vignali DA: The development and function of regulatory T cells. Cell Mol Life Sci 66: 2603-2622, 2009.

12. Shalev I, Schmelzle M, Robson SC and Levy G: Making sense of regulatory $\mathrm{T}$ cell suppressive function. Semin Immunol 23: 282-292, 2011.

13. Leng FY, Liu JL, Liu ZJ, Yin JY and Qu HP: Increased proportion of CD4(+)CD25(+)Foxp3(+) regulatory $\mathrm{T}$ cells during the early-stage sepsis in ICU patients. J Microbiol Immunol Infect: Aug 23, 2012 (Epub ahead of print).

14. Monneret G, Debard AL, Venet F, et al: Marked elevation of human circulating $\mathrm{CD} 4{ }^{+} \mathrm{CD} 25^{+}$regulatory $\mathrm{T}$ cells in sepsis-induced immunoparalysis. Crit Care Med 31: 2068-2071, 2003.

15. Saito K, Wagatsuma T, Toyama H, et al: Sepsis is characterized by the increases in percentages of circulating $\mathrm{CD} 4{ }^{+} \mathrm{CD} 25^{+}$regulatory $\mathrm{T}$ cells and plasma levels of soluble CD25. Tohoku J Exp Med 216: 61-68, 2008. 
16. Heuer JG, Zhang T, Zhao J, et al: Adoptive transfer of in vitro-stimulated $\mathrm{CD} 4^{+} \mathrm{CD} 25^{+}$regulatory $\mathrm{T}$ cells increases bacterial clearance and improves survival in polymicrobial sepsis. J Immunol 174: 7141-7146, 2005.

17. Scumpia PO, Delano MJ, Kelly KM, et al: Increased natural $\mathrm{CD} 4{ }^{+} \mathrm{CD} 25^{+}$regulatory $\mathrm{T}$ cells and their suppressor activity do not contribute to mortality in murine polymicrobial sepsis. J Immunol 177: 7943-7949, 2006.

18. Wisnoski N, Chung CS, Chen Y, Huang X and Ayala A: The contribution of $\mathrm{CD} 4{ }^{+} \mathrm{CD} 25^{+} \mathrm{T}$-regulatory-cells to immune suppression in sepsis. Shock 27: 251-257, 2007.

19. Porwoll JM, Gebel HM, Rodey GE and Markham RB: In vitro response of human T cells to Pseudomonas aeruginosa. Infect Immun 40: 670-674, 1983.

20. Lee N, Ahn B, Jung SB, Kim YG, Kim H and Park WJ: Conformation-dependent antibody response to Pseudomonas aeruginosa outer membrane proteins induced by immunization in humans. FEMS Immunol Med Microbiol 27: 79-85, 2000.

21. MuXY: Success in establishing the MSHA-positive Pseudomonas aeruginosa fimbrial strain. Wei Sheng Wu Xue Bao 26: 176-179, 1986 (In Chinese).

22. Jia L, Wang C, Kong H, et al: Effect of PA-MSHA vaccine on plasma phospholipids metabolic profiling and the ratio of Th2/Th1 cells within immune organ of mouse IgA nephropathy. J Pharm Biomed Anal 43: 646-654, 2007.
23. Wu Y, Borde M, Heissmeyer V, et al: FOXP3 controls regulatory $\mathrm{T}$ cell function through cooperation with NFAT. Cell 126: 375-387, 2006.

24. Mallat Z, Besnard S, Duriez M, et al: Protective role of interleukin-10 in atherosclerosis. Circ Res 85: e17-e24, 1999.

25. Pinderski Oslund LJ, Hedrick CC, Olvera T, et al: Interleukin-10 blocks atherosclerotic events in vitro and in vivo. Arterioscler Thromb Vasc Biol 19: 2847-2853, 1999.

26. Ait-Oufella H, Taleb S, Mallat Z and Tedgui A: Cytokine network and $\mathrm{T}$ cell immunity in atherosclerosis. Semin Immunopathol 31: 23-33, 2009.

27. Davidson NJ,Leach MW,Fort MM, et al: Thelper cell 1-type CD4 ${ }^{+}$ $\mathrm{T}$ cells, but not $\mathrm{B}$ cells, mediate colitis in interleukin 10-deficient mice. J Exp Med 184: 241-251, 1996.

28. Damas P, Reuter A, Gysen P, Demonty J, Lamy M and Franchimont P: Tumor necrosis factor and interleukin-1 serum levels during severe sepsis in humans. Crit Care Med 17: 975-978, 1989.

29. Girardin E, Grau GE, Dayer JM, Roux-Lombard P and Lambert PH: Tumor necrosis factor and interleukin-1 in the serum of children with severe infectious purpura. N Engl J Med 319: 397-400, 1988. 